\title{
Estrutura fitossociológica do manguezal da Lagoa do Roteiro, Alagoas, Nordeste do Brasil
}

\author{
Phytosociological structure of mangrove in Roteiro Lagoon, Alagoas state, \\ Northeastern Brazil
}

\author{
Simony Andrade Silva ${ }^{\mathrm{I}}$, Vitória Barros ${ }^{\mathrm{I}}$, Alexandre Oliveira ${ }^{\mathrm{II}}$
}

\begin{abstract}
Resumo
Este estudo teve como objetivo caracterizar a estrutura do manguezal da Lagoa do Roteiro no estado de Alagoas, através de parâmetros fitossociológicos. Para isso, foram traçados seis transectos, cada um dividido em três parcelas de $400 \mathrm{~m}^{2}$, distanciadas 10 metros entre si. Para a verificação dos fatores abióticos foram utilizados termômetro (temperatura) e refratômetro (salinidade). Foram coletadas ainda três amostras de sedimento, a uma profundidade de $20 \mathrm{~cm}$, para a análise da granulometria e matéria orgânica. Foram mensuradas 449 árvores, apresentando árvores de Rhizophora mangle, Laguncularia racemosa e Avicennia schaueriana. A densidade média da vegetação arbórea foi de 624 ind.ha ${ }^{-1}$, sendo a espécie Rhizophora mangle a que apresentou os maiores índices fitossociológicos, com valor de importância de $220,59 \%$. Os valores dos parâmetros fitossociológicos apresentaram grande amplitude nas parcelas analisadas, ocorrendo desde plântulas até árvores adultas. Os valores de temperatura não apresentaram diferenças significativas entre as médias das parcelas franja, intermediária e interior, porém a salinidade se destacou, apresentando um aumento em relação ao interior do bosque. Houve predominância de sedimento areno-lodoso no manguezal estudado. Dessa maneira, a análise fitossociológica revelou elevada diversidade estrutural, em um ambiente onde os fatores abióticos contribuem para o estabelecimento das espécies arbóreas, e uma elevada contribuição da espécie Rhizophora mangle em todos os parâmetros fitossociológicos avaliados no ecossistema.
\end{abstract}

Palavras-chave: Bosque de mangue; Fitossociologia; Parâmetros abióticos

\begin{abstract}
This study aimed to characterize the phytosociological structure of the mangrove in Roteiro Lagoon in the state of Alagoas, through phytosociological parameters. For that purpose, six transects were traced, divided into three $400 \mathrm{~m}^{2}$ plots each, 10 meters apart each other. To check the abiotic factors, a thermometer (temperature) and a refractometer (salinity) were used. Three sediment samples were also collected, at a depth of $20 \mathrm{~cm}$, for the granulometry and organic matter analyses. A total of 449 trees were measured, showing trees Rhizophora mangle, Laguncularia racemosa and Avicennia schaueriana. The average density of the arboreal vegetation was 624 ind.ha ${ }^{-1}$, with the Rhizophora mangle species showing the highest phytosociological indexes, with a value of $220.59 \%$. The values of the phytosociological parameters showed great amplitude in the analyzed plots, occurring from seedlings to adult trees. The temperature values did not show significant differences between the average of the fringe, intermediate and interior portion, however, the salinity stood out, showing an increase in relation to the interior of the forest. There was a predominance of sandy silt in the studied mangrove forest. Thus, the phytosociological analysis revealed high structural diversity, in an environment where the abiotic factors contribute to the establishment of tree species and a high contribution of the Rhizophora mangle species in all phytosociological parameters assessed in the ecosystem.
\end{abstract}

Keywords: Mangrove forest; Phytosociology; Abiotic parameters

Engenheira de Pesca, Bacharela, Unidade Educacional Penedo, Universidade Federal de Alagoas, Av. Beira Rio, s/n, CEP 57200-000, Penedo (AL), Brasil. simonyeng.pesca@hotmail.com (ORCID: 0000-0002-1107-6647) / vitoriapetra@gmail.com (ORCID: 0000-0001-9826-3631)

II Biólogo, Dr., Professor da Unidade Educacional Penedo, Universidade Federal de Alagoas, Av. Beira Rio, s/n, CEP 57200-000, Penedo (AL), Brasil. alexandre.oliveira@penedo.ufal.br (ORCID: 0000-0002-9076-3524) 


\section{Introdução}

O Manguezal é um ecossistema costeiro característico de áreas onde há o encontro dos ambientes terrestre e marinho, comum de regiões com climas tropicais e subtropicais e sujeito ao regime das marés (SCHAEFFER-NOVELLI; VALE; CINTRÓN, 2015). Globalmente, os manguezais ocupam uma área de cerca de $137.760 \mathrm{~km}^{2}$, distribuídos em 118 países e territórios. Essa estimativa diminui com o aumento da latitude, exceto entre $20^{\circ}$ e $25^{\circ}$ de latitude norte, onde se localizam os Sundarbans, a maior área de manguezais do mundo, localizado na divisa entre o estado indiano de Bengala Ocidental e Bangladesh (GIRI et al., 2011). Os levantamentos mais recentes sobre a área de cobertura no Brasil variam de 962.683 a 1,071,083.74 ha, o que representa cerca de 7,0 a 8,9\% do total mundial da área de ocorrência desse ecossistema (BRAIL, 2010; MAGRIS; BARRETO, 2010; GIRI et al., 2011). Os manguezais podem ser encontrados em quase todo o litoral brasileiro, desde o Oiapoque $\left(04^{\circ} 30^{\prime} \mathrm{N}\right)$ até a Lagoa de Santo Antônio (28 $\left.28^{\circ} \mathrm{S}\right)$ (SOARES et al., 2012).

Em Alagoas, os manguezais ocorrem ao longo de todas as áreas estuarinas, ao longo dos rios, canais e junto aos corpos de água das principais lagunas costeiras (CORREIA; SOVIERZOSKI, 2009). Em razão de ser um ambiente costeiro, torna-se o habitat de diversas populações e possui condições ideais para a reprodução de várias espécies de peixes, crustáceos e moluscos, sendo considerado um dos ambientes naturais mais produtivos do Brasil (BERNINI et al., 2014; BLOTTA et al., 2016).

Com relação à vegetação, nota-se que há baixa diversidade, uma vez que poucas espécies conseguem se adaptar às condições de maré, salinidade, substrato inconsolidado e pouco oxigenado (OLIVEIRA; TOGNELLA, 2014). Nos manguezais brasileiros ocorrem três gêneros, Rhizophora, Laguncularia e Avicennia com um total de seis espécies: Rhizophora mangle, Rhizophora harrisonii, Rhizophora racemosa, Laguncularia racemosa, Avicennia schaueriana, Avicennia germinans (INSTITUTO CHICO MENDES DE CONSERVAÇÃO E BIODIVERSIDADE, 2018).

O manguezal pode ser estruturado como um continuum de feições de acordo com as características específicas existentes em cada zona, classificadas como apicum, bosque de mangue e lavado (SCHAEFFER-NOVELLI; VALE; CITRÓN, 2015). Essas regiões exercem influência na formação da vegetação e, geralmente, as espécies vegetais de mangue estão distribuídas em zonas em relação à linha d'água, constituindo em uma fisionomia peculiar (ALMEIDA et al., 2014).

A trajetória da dinâmica da vegetação de mangue pode mudar através do tempo com as mudanças do nível relativo do mar. Tanto por mudanças no uso e ocupação de uma determinada bacia que tem no seu nível de base florestas de mangue em pleno desenvolvimento, quanto pelas mudanças climáticas locais e/ou regionais (microescala e mesoescala), os manguezais constituem excelentes bioindicadores, respondendo aos gradientes de inundação, de nutrientes e de salinidade (SCHAEFFER-NOVELLI; VALE; CINTRÓN, 2015). Tal heterogeneidade parece ser uma resposta à interação de vários fatores abióticos (fatores edáficos e salinidade) e antrópicos que atuam em diferentes escalas espaciais e temporais (BERNINI; REZENDE, 2010).

De acordo com o exposto, a pesquisa foi desenvolvida a partir da elaboração de questionamentos sobre o tema, são eles: Qual a influência dos fatores abióticos no crescimento das árvores do manguezal? Como a concentração de matéria orgânica e o tipo de sedimento podem influenciar no estabelecimento de uma espécie arbórea? E por fim, qual a espécie arbórea que mais se destaca na região? 


\section{Material e método}

O estudo foi realizado na Reserva Ecológica de Manguezais da Lagoa do Roteiro, associado à desembocadura do Rio São Miguel, entre os municípios de Roteiro e Barra de São Miguel (S 1950' 13,50"; W 3555’40,09”) com área total de 742 hectares (Figura 1). Essa reserva foi criada pelo Decreto no 32.355/1987, com objetivo da preservação integral do meio natural, sendo limitadas todas as interferências sobre esse ecossistema, sendo permitida apenas atividades como a pesca tradicional e coleta de moluscos e outros recursos pesqueiros (INSTITUTO DO MEIO AMBIENTE, 2019). Para o desenvolvimento do estudo foram delimitados seis transectos no manguezal, sendo três transectos na comunidade de Palatéia (A, B e C), localizado na margem de Barra de São Miguel; dois transectos em ilhas localizadas na lagoa (E e F) e um transecto próximo a cidade de Roteiro (D).

\section{Figura 1 - Distribuição dos transectos no manguezal da Lagoa do Roteiro, Alagoas}

Figure 1 - Transects distribution in Roteiro Lagoon mangrove, Alagoas state

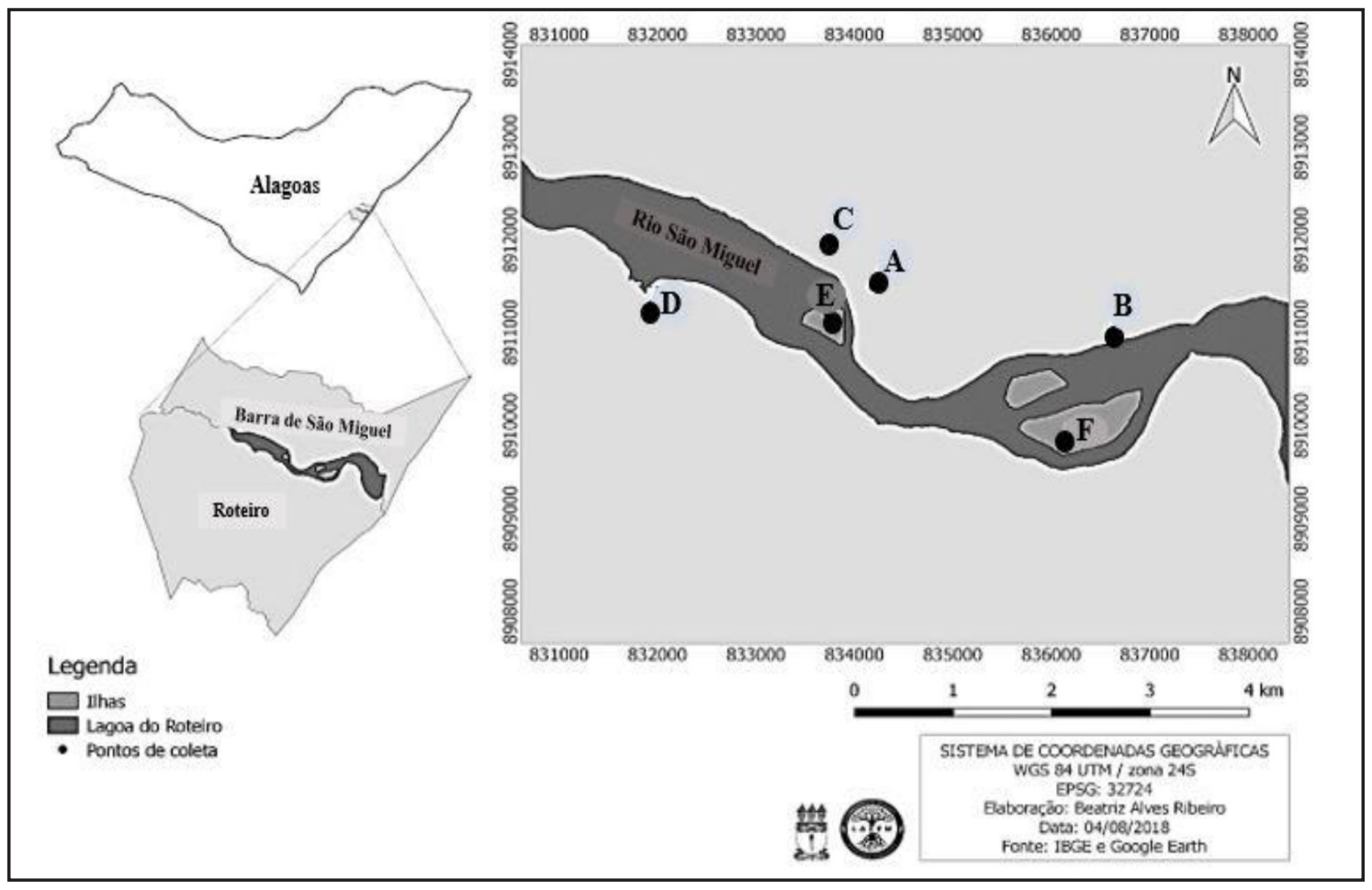

Fonte: Autores (2019)

No litoral alagoano o vento predominante é o vento nordeste nos meses de verão e o vento sul nos meses de inverno, o qual favorece a formação de chuvas (CORREIA; SOVIERZOSKI, 2009). Com base nas características climáticas e de acordo com a classificação de "Köppen”, o litoral alagoano é representado pelo tipo As, que corresponde ao clima quente e úmido (Tropical e Equatorial), com um período de chuvas determinado, ocorrendo entre os meses de inverno e consequentemente com verão seco (SALLES, 1995; ALVARES et al., 2014).

O estudo da fitossociologia do bosque seguiu a metodologia proposta por Cintrón e Schaeffer-Novelli (1984), que é baseada nas informações estruturais coletadas no bosque em estudo, método denominado de parcelas múltiplas. Para cada transecto de estudo, foram demarcados grupos de três parcelas, denominadas franja, intermediária e interior. A parcela 
denominada franja, corresponde à zona do bosque de mangue mais próxima do canal estuarino e sob maior influência da variação de maré; intermediária correspondente à zona média do bosque e parcela interior caracterizada por ser a zona mais distante da linha d'água, cada parcela medindo $400 \mathrm{~m}^{2}$, distanciados em $10 \mathrm{~m}$ entre si, perpendiculares à margem do rio.

As coletas foram realizadas bimestralmente durante o período de um ano, sendo as árvores identificadas quanto à espécie. Para cada espécie arbórea viva foram anotadas a altura, por estimativa visual, e a circunferência com o auxílio de uma fita métrica para obter o Perímetro na Altura do Peito (PAP), sendo posteriormente transformado para Diâmetro na Altura do Peito (DAP). Foram considerados todos os indivíduos com DAP maior ou igual a $2,5 \mathrm{~cm}$.

O cálculo dos parâmetros estruturais mencionados por parcela, espécie e classe de diâmetro, seguiu a metodologia de Schaeffer-Novelli e Cintrón, (1986). Foram calculados o Diâmetro da Altura do Peito (DAP); Diâmetro da Altura do Peito Médio (DAP); Dominância Relativa (DoR); Densidade Relativa (DR); Frequência Absoluta (FA); Frequência Relativa (FR); Índice de Valor de Importância (IVI).

Para visualizar as diferenças entre as áreas (franja, intermediária e interior) em relação aos parâmetros fitossociológicos da vegetação arbórea, os dados foram analisados através da estatística descritiva, utilizando a análise de variância ANOVA $(\mathrm{p}<0,05)$, com auxílio do programa BIOESTAT 5.0. O contraste entre as médias foi avaliado pelo teste de Tukey.

As análises dos fatores abióticos foram realizadas mensalmente com o auxílio de um termômetro, que foi utilizado para a verificação da temperatura do ar; do sedimento e da água intersticial e refratômetro para a salinidade. Para isso, foram instalados durante a maré baixa, em cada parcela um tubo de PVC (15 $\mathrm{cm}$ de profundidade), para a determinação da temperatura da água intersticial e da salinidade.

Para o estudo da granulometria e matéria orgânica foram coletadas bimestralmente três amostras de sedimento em cada parcela do transecto, com o auxílio de um coletor (tipo "corer") de PVC, inserido manualmente no sedimento, a uma profundidade de $20 \mathrm{~cm}$, sendo que cada amostra continha aproximadamente $200 \mathrm{~g}$. O sedimento foi colocado na estufa onde permaneceu durante 24 horas, a uma temperatura de $80^{\circ} \mathrm{C}$. O método para a determinação das classes texturais foi o peneiramento a seco, onde as amostras de cada uma das parcelas foram pesadas e colocadas em um agitador de peneiras granulométricas durante 15 minutos. As classificações texturais seguiram a metodologia de Shepard (SUGUIO, 1973), baseada nas porcentagens de areia, silte e argila. Para isso, foi utilizado o programa SYSGRAN 3.0 (Sistema de Análises Granulométricas).

A matéria orgânica foi analisada através de amostras de 20 gramas de sedimento, que após secas foram submetidas à incineração em forno Mufla a uma temperatura de $550^{\circ} \mathrm{C}$ por aproximadamente 5 horas. O percentual de matéria orgânica foi obtido através de diferença entre peso inicial e final (SUGUIO, 1973).

\section{Resultados e discussão}

Foram mensuradas 449 árvores, apresentando 360 indivíduos de Rhizophora mangle L., 71 indivíduos de Laguncularia racemosa (L.) Gaertn. f. e 18 indivíduos de Avicennia schaueriana Stapf e Leechm. ex Moldenke. As árvores localizadas no manguezal da Lagoa do Roteiro, também foram encontradas em outras regiões, como no manguezal da zona Portuária de São Luís - Maranhão (GONÇALVES et al., 2018); no manguezal do Rio Coruripe - Alagoas (RODRIGUES, 2015); no estuário do Espírito Santo - ES (LONDE et al., 2013); no manguezal do estuário do Rio das Ostras - RJ (BERNINE et al., 2014); em São Paulo (BLOTTA et al., 2016) e no Paraná (MADI et al., 2016).

$\mathrm{O}$ manguezal apresentou densidade média das árvores de 624 ind.ha $^{-1}$. Com relação à densidade relativa foi de $80,18 \%$ para Rhizophora mangle, $15,81 \%$ para Laguncularia racemosa e 4,01\% para Avicennia schaueriana. A espécie Rhizophora mangle apresentou frequência de 54,55\%, dominância de $85,87 \%$ e valor de importância de 220,59\%. Laguncularia racemosa apresentou valores de frequência de $30,30 \%$, dominância de $9,90 \%$ e valor de importância de 56,01\%. Já 
Avicennia schaueriana apresentou frequência, dominância e valor de importância, de 15,15\%, $4,23 \%$ e $23,39 \%$, respectivamente.

Resultados semelhantes foram encontrados por Paraguassu e Silva (2007), em Porto do Sauípe - Bahia, onde Rhizophora mangle apresentou os maiores índices fitossociológicos para todos os parâmetros analisados, seu índice de importância foi de $244,92 \%$, seguida por Laguncularia racemosa com 55\%. Em um estudo realizado por Rodrigues (2015), no manguezal do Rio Coruripe - Alagoas, verificou-se que Rhizophora mangle exibiu uma frequência de $74,08 \%$, dominância de $75,91 \%$, valor de importância de $224,24 \%$, enquanto Laguncularia racemosa apresentou frequência de $25,92 \%$, dominância de $24,09 \%$, valor de importância de $75,76 \%$. Neves (2014) verificou que na região do Baixo são Francisco a espécie Rhizophora mangle destacou-se com o maior índice de valor de importância de 61,35\%, enquanto Laguncularia racemosa apresentou 38,65\%. Em ambos os estudos a espécie Rhizophora mangle apresentou os maiores índices em todos os parâmetros analisados, característica semelhante encontrada no presente estudo.

O manguezal da Lagoa do Roteiro apresentou bosques com parâmetros fitossociológicos distintos, onde a menor altura média das árvores foi de 3,0 $\pm 0,98 \mathrm{~m}$ na região franja do transecto $B$, fato que pode ser explicado por ter sido observada nessa parcela a presença de resíduos sólidos. Essa característica indica que a parcela sofreu impactos antrópicos. Segundo Cintrón e Schaeffer-Novelli (1984), a ação dos tensores antrópicos associados aos naturais, que agem sobre esse ecossistema, restringe o desenvolvimento do bosque, interferindo tanto nas características estruturais como na sua diversidade funcional.

A região interior do transecto $C$ atingiu a maior altura média $(18,75 \pm 10,17 \mathrm{~m})$ sendo que essa parcela apresentou características ambientais que favoreceram o desenvolvimento das árvores, como por exemplo, elevada concentração de matéria orgânica (Figura 2).

Figura 2 - Altura (média \pm desvio padrão) das parcelas analisadas entre agosto de 2016 a julho de 2017, no manguezal da Lagoa do Roteiro, Alagoas

Figure 2 - Height (average \pm standard deviation) of the plots analyzed between August 2016 and July 2017, in Roteiro Lagoon mangrove, Alagoas state

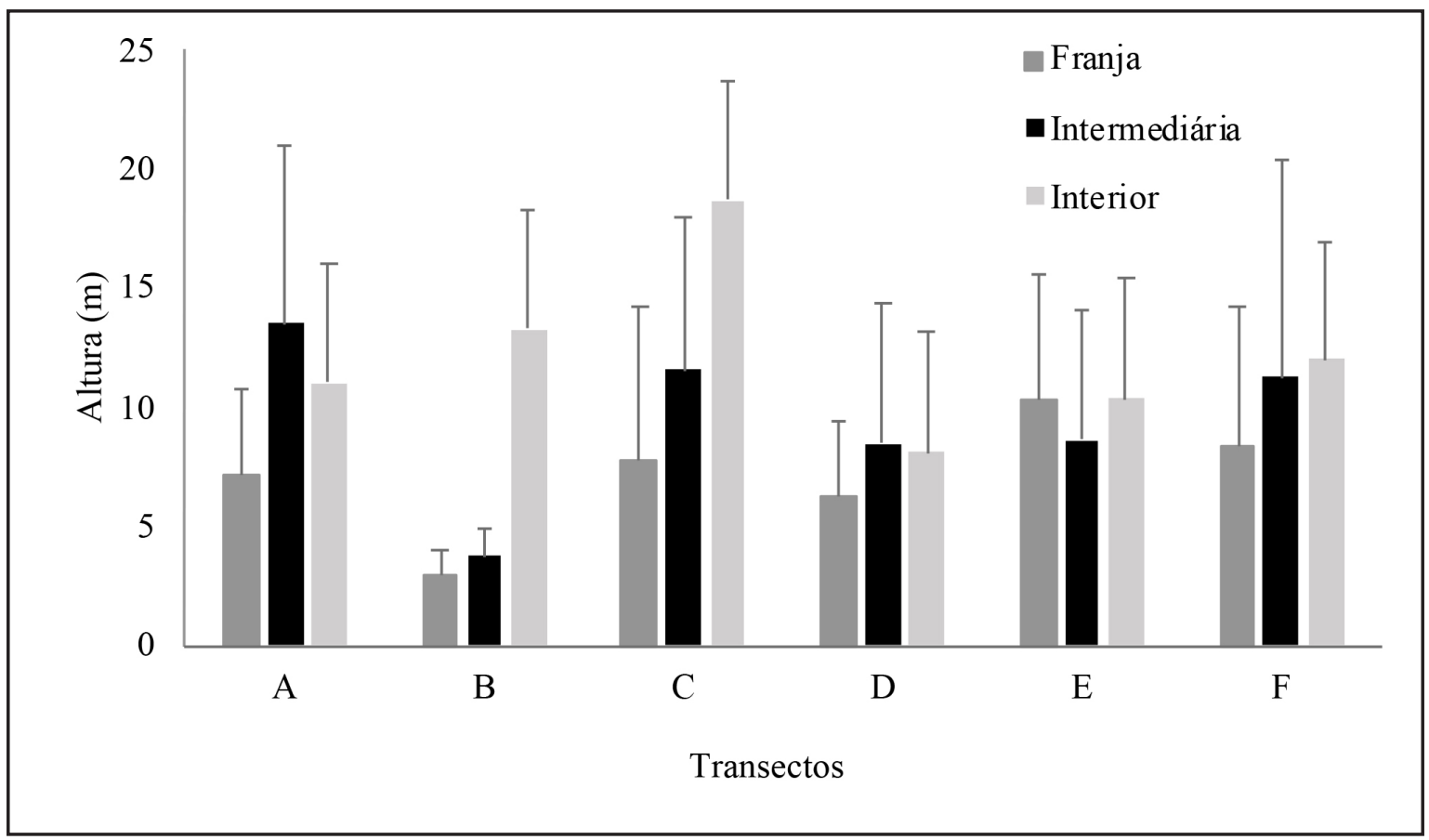

Fonte: Autores (2019) 
Com relação ao DAP médio, as parcelas apresentaram valores que variaram de 4,79 $\mathrm{cm}$ (franja do transecto B) a 21,63 cm (interior do transecto C). Esses valores indicam bosques com grande heterogeneidade da flora, pois indicam a ocorrência de árvores jovens e adultas. As parcelas franja e intermediária do transecto $\mathrm{B}$ apresentaram os menores valores médios, estatisticamente significativos $(\mathrm{p} \leq 0,05)$, em relação aos demais transectos (Figura 3). Esse transecto também apresentou grande quantidade de plântulas. Esse fato pode estar relacionado diretamente à ações e impactos antrópicos, visto que é o transecto mais próximo ao acesso para as comunidades ribeirinhas e vias de tráfego de escoamento de produção.

Figura 3 - DAP médio (média \pm desvio padrão) das parcelas analisadas entre agosto de 2016 a julho de 2017, no manguezal da Lagoa do Roteiro, Alagoas

Figure 3 - Average DBP (average \pm standard deviation) of the plots analyzed between August 2016 and July 2017, in Roteiro Lagoon mangrove, Alagoas state

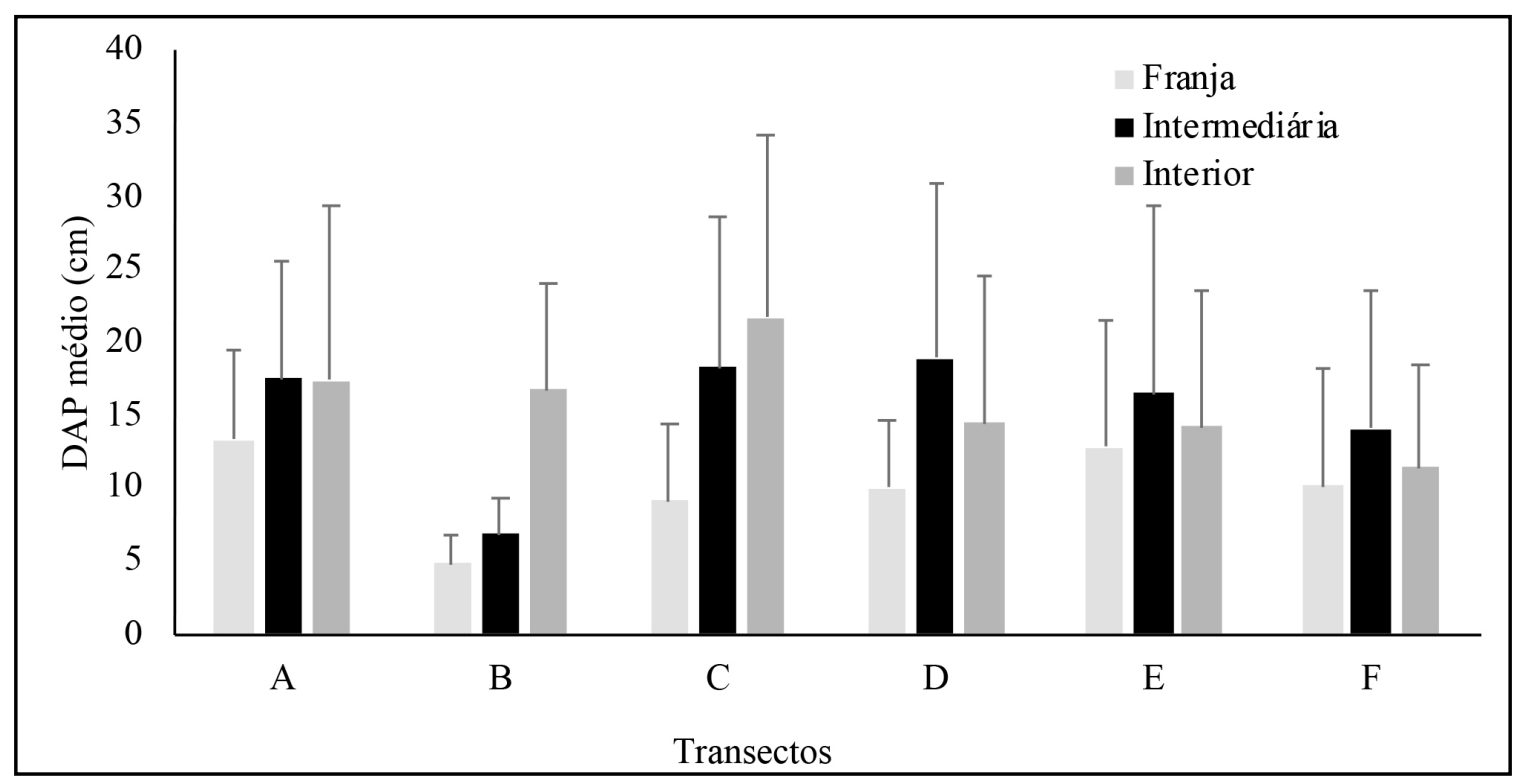

Fonte: Autores (2019)

Valores semelhantes foram encontrados por Santos (2013), no manguezal do São Francisco, onde foi observado que a altura média das árvores variou entre 2,88 a 15,63 m e o diâmetro médio apresentou variação de 3,95 a $19,74 \mathrm{~cm}$, demonstrando um desenvolvimento estrutural heterogêneo entre os bosques, resultado semelhante ao encontrado no manguezal da Lagoa do Roteiro.

Almeida et al. (2014) afirmam que tais características ocorrem nos diferentes ambientes de floresta, pois os manguezais são dinâmicos, possuindo crescimento contínuo e constantemente interferindo diretamente no seu restabelecimento e renovação.

Os parâmetros abióticos analisados, tanto da temperatura da água; do sedimento e do ar não apresentaram diferenças significativas entre as médias das parcelas franja, intermediária e interior (Tabela 1). 


\section{Tabela 1 - Parâmetros abióticos (média \pm desvio padrão) das parcelas (franja, intermediária e interior), analisados entre agosto de 2016 a julho de 2017 no Manguezal da Lagoa do Roteiro, Alagoas}

Table 1 - Abiotic parameters (average \pm standard deviation) of the plots (fringe, intermediate and interior), analyzed between August 2016 and July 2017, in Roteiro Lagoon mangrove,

Alagoas state

\begin{tabular}{lcccc}
\hline \multirow{2}{*}{ Parcela } & \multicolumn{3}{c}{ Temperatura $\left({ }^{\circ} \mathbf{C}\right)$} & \multirow{2}{*}{ Salinidade (\%o) } \\
\cline { 2 - 4 } & Água & Sedimento & Ar & \\
\hline Franja & $26,63 \pm 2,21$ & $26,83 \pm 2,04$ & $29,06 \pm 1,78$ & $10,00 \pm 9,85$ \\
Intermediária & $27,00 \pm 2,45$ & $26,92 \pm 1,69$ & $29,19 \pm 2,56$ & $13,02 \pm 9,35$ \\
Interior & $26,50 \pm 1,73$ & $26,83 \pm 1,94$ & $28,94 \pm 2,11$ & $20,60 \pm 8,20$ \\
\hline
\end{tabular}

Fonte: Autores (2019)

Os resultados de salinidade da água intersticial apresentaram valores distintos, onde foi possível observar um aumento da salinidade em direção ao interior do bosque. Resultados semelhantes foram observados por Estrada et al. (2013). Isso ocorre devido à ação das marés que variam ao longo do manguezal, isto é, algumas áreas são inundadas diariamente enquanto outras são atingidas apenas sazonalmente pelas marés de sizígia (SCHAEFFER-NOVELLI, 1995). Sendo assim, a região interior do bosque é banhada somente pelas marés de maior amplitude, gerando uma região com maior acúmulo de sais. Segundo Estrada et al. (2008), esse padrão de aumento da salinidade em direção ao interior do bosque, provavelmente, é determinado pelo efeito combinado da redução das inundações das marés e da frequência de descarga com a precipitação anual e evaporação, fazendo com que esses locais tenham valores de salinidade mais elevados.

A distribuição das espécies de plantas pelas zonas formadas depende de como funcionam seus sistemas de excreção de sal e sustentação e do quanto as es pécies conseguem moldá-los entre as zonas (NASCIMENTO et al., 2008). No manguezal estudado a salinidade não condicionou a zonação da espécie Rhizophora mangle, pois a mesma esteve presente em diferentes concentrações de salinidade e em todas as parcelas.

$\mathrm{Na}$ análise sedimentológica do material coletado ocorreu a predominância de sedimento areno-lodoso, com uma classificação de areia média e areia fina em todos os pontos coletados, obtendo um total de $92,44 \%$. Os sedimentos finos foram predominantes, assim como a presença de silte e argila com 5,38\%. Já a fração cascalho foi a menos significativa com valor de $2,28 \%$. Em um estudo realizado por Silva (2001), a fração silte grossos foram encontrados no estuário do Rio São Miguel, até a cidade de Roteiro e mais na parte interna do sistema estuarino lagunar, próximo às ilhas de mangues e próximo a praia do Gunga - AL. Tais características evidenciam que o manguezal analisado apresenta uma elevada concentração de sedimentos finos característicos desse ecossistema.

A concentração de matéria orgânica durante o estudo apresentou valores elevados entre os transectos (B); (C); (D) e (F),obtendo uma concentração equivalente respectivamente a 58,7\%; $70 \% ; 60,4 \%$ e $58,2 \%$, fato que pode ser explicado pelo próprio ecossistema produzir uma elevada quantidade de matéria orgânica de forma natural através dos organismos (Figura 4). Os pontos (A) e (E) apresentaram um percentual menor se comparado aos demais pontos, o que pode estar sendo influenciado pela presença de sedimentos mais finos como silte e areia muito fina. Espécies de manguezal, principalmente aquelas pertencentes à espécie Rhizophora mangle, acumulam maior teor de matéria orgânica (TWILLEY et al.,1995). Fato que pode estar relacionado por Rhizophora mangle ser encontrada em todos os pontos analisados na Lagoa do Roteiro. 
Figura 4 - Porcentagem (média \pm desvio padrão) de Matéria Orgânica das parcelas analisadas entre agosto de 2016 a julho de 2017, no Manguezal da Lagoa do Roteiro, Alagoas

Figure 4 - Percentage (average \pm standard deviation) of organic matter of the plots analyzed between August 2016 and July 2017, in Roteiro Lagoon mangrove, Alagoas state

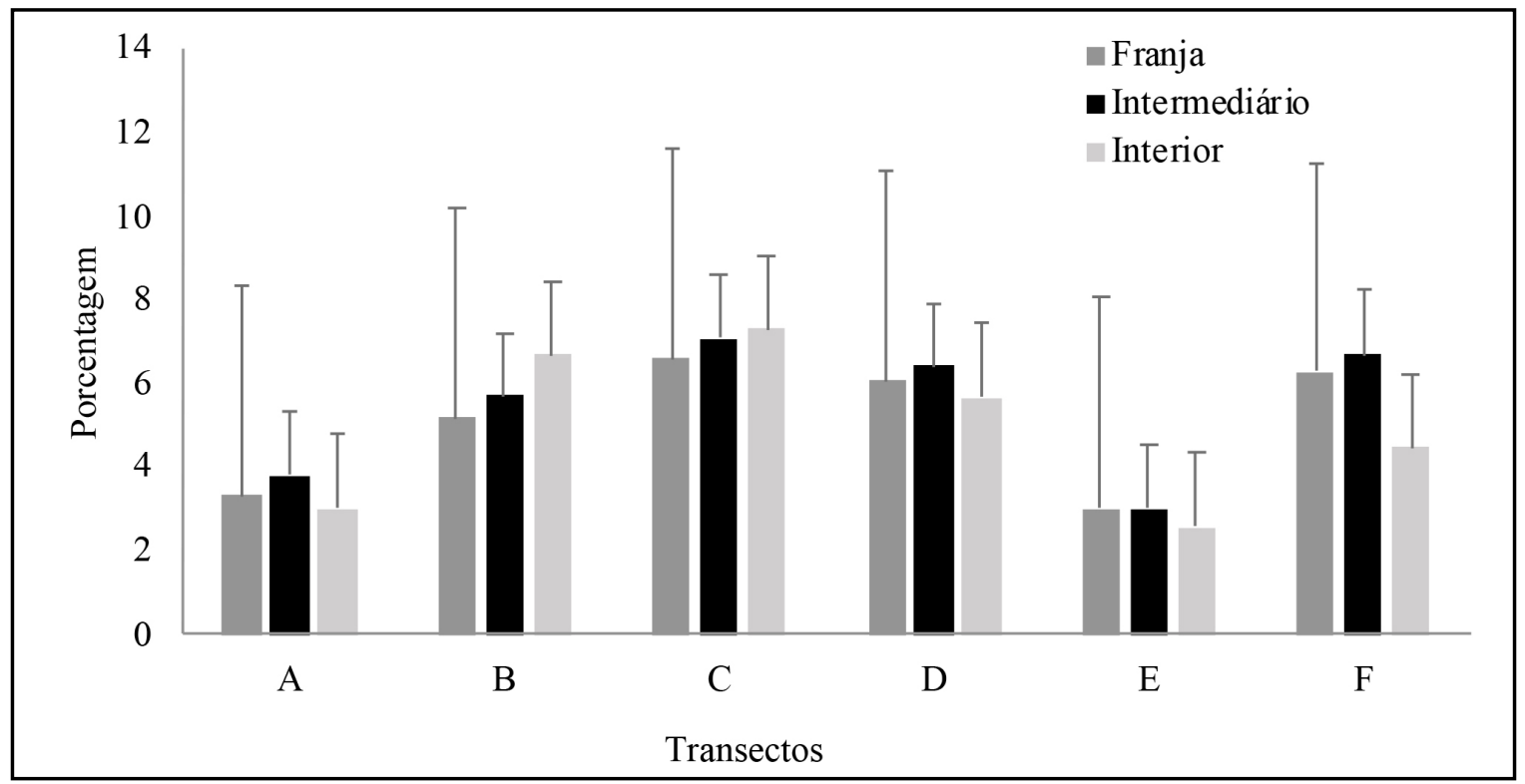

Fonte: Autores (2019)

As características fitossociológicas das parcelas variaram entre os bosques. Tais variações foram estatisticamente significativas para o parâmetro DAP, entre as parcelas Franja e Intermediário $(\mathrm{p} \leq 0,05)$. Essas diferenças encontradas entre as parcelas Franja e Intermediária dos transectos podem estar relacionadas às marés, quando estes ambientes sofrem modificações em relação às características estruturais do bosque, sendo que a parcela Interior seria menos influenciada pelas marés produzindo parcelas mais homogêneas.

Tais características demonstraram uma grande variabilidade entre as parcelas, fato que pode estar relacionado aos fatores ambientais distintos em cada transecto estudado. Segundo Bernini e Rezende (2010), a heterogeneidade das características estruturais das florestas está em função das variações das energias subsidiárias, como marés e ondas, nutrientes, aporte de água doce, temperatura, vento, radiação solar e precipitação. Sendo assim, o melhor desenvolvimento estrutural observado na região reflete as boas condições ambientais para o desenvolvimento das florestas, particularmente no que se referem à disponibilidade adequada de água doce, nutrientes e luminosidade (BERNINI; REZENDE, 2010).

Assim, estudos fitossociológicos de uma floresta representam o passo inicial para o seu conhecimento, pois quando associados à sua dinâmica, pode-se construir uma base teórica para subsidiar a preservação e o uso de recursos da flora, a conservação de ecossistemas similares e a recuperação de áreas ou fragmentos florestais degradados, contribuindo substancialmente para seu manejo (ARRUDA; DANIEL, 2007). O gerenciamento de áreas de manguezais deve ser considerado juntamente com os custos de oportunidade em recreação, turismo, pescaria, educação e pesquisa, os quais são oferecidos, sem necessitar a manipulação do sistema ambiental, conservando assim os fluxos de matéria e nutrientes (energia) que sustentam sua produção e a qualidade da água na zona costeira (CUNHA-LIGNON et al., 2011). 


\section{Conclusão}

No manguezal da Lagoa do Roteiro ocorreu predominância de Rhizophora mangle para todos os parâmetros avaliados. A espécie Laguncularia racemosa foi a segunda mais importante, seguida por Avicennia schaueriana. O estudo demonstrou variabilidade dos parâmetros fitossociológicos entre as parcelas, fato que pode estar relacionado aos fatores ambientais distintos em cada transecto estudado. O valor de temperatura do ecossistema analisado não apresentou diferenças significativas entre as médias das parcelas, porém o parâmetro salinidade obteve um aumento considerável em direção ao interior do bosque. O sedimento foi classificado como areno-lodoso com elevada concentração de matéria orgânica, as características encontradas durante o estudo foram semelhantes às condições ambientais da região. $O$ estudo da estrutura do manguezal é muito importante para o conhecimento da área de estudo e serve como ferramenta de medidas de manejo para a conservação desses ambientes, além de servir como subsídio para futuros estudos.

\section{Referências}

ALMEIDA, V. C. et al. Caracterização estrutural do manguezal do rio Tabatinga, Suape, Pernambuco. Tropical Oceanography, Recife, v. 42, n. 1, p. 33-47, 2014.

ALVARES, C. A. et al. Köppen's climate classification map for Brazil. Meteorologische Zeitschrift, Karlsruhe, v. 22, n. 6, p. 711-728, 2014.

ARRUDA, L.; DANIEL, O. Florística e diversidade em um fragmento de floresta estacional semidecidual aluvial em Dourados, MS. Revista Floresta, Curitiba, v. 37, n. 2, p. 187-199, 2007.

BERNINI, E. et al. Fitossociologia de florestas de mangue plantadas e naturais no estuário do Rio das Ostras, Rio de Janeiro, Biotemas, São Carlos, v. 27 n. 1, p. 37-48, 2014.

BERNINI, E.; REZENDE, C. E. Variação estrutural em florestas de mangue do estuário do rio Itabapoana, ES-RJ. Biotemas, São Carlos, v. 23, n. 1, p. 49-60, 2010.

BLOTTA, K. D. et al. Fitossociologia comparativa de dois manguezais: Canal de Bertioga/SP e do estuário da Barra do Rio Una-Peruíbe-SP. UNISANTA Bioscience Journal, Uberlândia, v. 5, n. 3, p. 271-282, 2016.

BRASIL. Ministério do Meio Ambiente. Gerência de Biodiversidade Aquática e Recursos Pesqueiros. Panorama da conservação dos ecossistemas costeiros e marinhos no Brasil. Brasília, 2010. 148 p.

CINTRÓN, G.; SCHAEFFER-NOVELLI, Y. Methods for studying mangrove structure. In: SNEDAKER, S. C.; SNEDAKER J. G. (ed.). The mangrove ecosystem: research methods. United Nations Educational, Scientific and Cultural Organization, Paris, p. 91-113, 1984.

CORREIA, M. D.; SOVIERZOSKI, H. H. Ecossistemas costeiros de Alagoas - The mangrove ecosystem: research methods. Bungay: UNESCO, 1984. 251 p. Brasil. Rio de Janeiro: Technical Books, 2009. 144 p.

CUNHA-LIGNON, M. et al. Mangrove forests submitted to depositional processes and salinity variation investigated using satellite images and vegetation structure surveys. Journal of Coastal Research, Coconut Creek, v. 1, p. 344-348, 2011.

ESTRADA, G.C. D.etal. Analysis of the structural variability of mangrove forests through the physiographic types approach. Aquatic Botany, Berlin, v. 111, p. 135-143, 2013.

ESTRADA, G. C. D. et al. Annual growth rings in the mangrove Laguncularia racemosa (Combretaceae). Trees, Berlin, v. 22, p. 663-670, 2008.

GIRI, C. et al. Status and distribution of mangrove forests of the world using earth observation satellite data. Global Ecology and Biogeography, New Jersey, v. 20, p. 154-159, 2011.

GONÇALVES, A. L. et al. Composição florística e fitossociológica do manguezal da zona portuária de São Luís, Maranhão, Brasil. Biofix Scientific Journal, Curitiba, v. 3, n. 1, p. 01-07, 2018. 
INSTITUTO CHICO MENDES DE CONSERVAÇÃO E BIODIVERSIDADE. Atlas dos Manguezais do Brasil. Brasília, 2018.

INSTITUTO DO MEIO AMBIENTE (AL). [Website]. Maceió, 2019. Disponível em http://www. meioambiente.al.gov.br. Acesso em: 06 jul. 2019.

LONDE, V. et al. Estrutura da vegetação de mangue associada ao gradiente de inundação no litoral norte do Espírito Santo, Revista Árvore, Viçosa, MG, v. 37, n. 4, p. 629-637, 2013.

MADI, A. P. L. M. et al. Estrutura do componente de regeneração natural e arbóreo de dois manguezais no estado do Paraná. Ciência Florestal, Santa Maria, v. 26, n. 1, p. 159-170, 2016.

MAGRIS, R. A.; BARRETO, R. Mapping and assessment of protection of mangrove habitats in Brazil. Pan-American Journal of Aquatic Sciences, Rio Grande, v. 5, n. 4, p. 546-556, 2010.

NASCIMENTO, E. R. et al. Distribuição de espécies arbóreas no gradiente de inundação no manguezal. In: MACHADO, G.; PRADO, P. I. K. L.; OLIVEIRA, A. A. (ed.). Livro do curso de campo "Ecologia da Mata Atlântica”. São Paulo: Universidade de São Paulo, 2008.

NEVES, M. A. Caracterização estrutural e serapilheira dos bosques de mangue da região estuarina do rio São Francisco (Baixo São Francisco). 2014. (Monografia) - Universidade Federal de Alagoas, Maceió, 2014.

OLIVEIRA, R. G.; TOGNELLA, M. M. P. Processo de colonização do manguezal do Rio Tavares por análise da estrutura de diferentes bosques. Brazilian Journal of Aquatic Science and Technology, Itajaí, v.18, n. 1, p. 9-18, 2014.

PARAGUASSU, L. A. A.; SILVA, M. N. Caracterização fitossociológica do manguezal de Porto de Sauipe, Entre Rios, Bahia. Diálogos e Ciência, Salvador, v. 5, n. 12, p. 1-12, 2007.

RODRIGUES, I. D. B. Caracterização estrutural do bosque de mangue do estuário do Rio Coruripe, Coruripe-Al. 2015. (Monografia) - Universidade Federal de Alagoas, Maceió, 2015.

SALLES, V. (org.). Guia do Meio Ambiente - Litoral de Alagoas. 3. ed. Maceió: Instituto do Meio Ambiente; Deutsche Gesellschaftfur Technische Zusammenarbeit; Fundação de Amparo à Pesquisa do Estado de Alagoas, 1995.

SANTOS, T. O. Florística, estrutura fitossociológica e produção de serapilheira do manguezal do Rio São Francisco. 2013. Dissertação (Mestrado) - Universidade Federal de Sergipe, Maceió, 2013.

SCHAEFFER-NOVELLI, Y.; CINTRÓN, G. Guia para estudo de áreas de manguezal: estrutura, função e flora. São Paulo: Caribbean Ecological Research, 1986. 150 p.

SCHAEFFER-NOVELLI, Y. Manguezal: ecossistema entre a terra e o mar. São Paulo: Caribbean Ecological Research, 1995. 64 p.

SCHAEFFER-NOVELLI, Y.; VALE, C.C.; CINTRÓN, G. Monitoramento do ecossistema manguezal: estrutura e características funcionais. In: TURRA, A.; DENADAI, M. R. (org.). Protocolos para o Monitoramento de Habitats Bentônicos Costeiros - Rede de Monitoramento de Habitats Bentônicos Costeiros - ReBentos. São Paulo: Instituto Oceanográfico da Universidade de São Paulo, 2015. p. 62-80.

SILVA, A. P. L. Estudos Geomorfológico e Sedimentológico do sistema Estuarino Lagunar do Roteiro - Alagoas. 2001. Dissertação (Mestrado) - Universidade Federal de Pernambuco, Recife, 2001.

SOARES, M. L. G. et al. Southern limit of the Western South Atlantic mangroves: assessment of the potential effects of global warming from a biogeographical perspective. Estuarine, Coastaland Shelf Science, Amsterdam, v. 101, p. 44-53, 2012.

SUGUIO, K. Introdução à sedimentologia. São Paulo: Edgar Blucher; EDUSP, 1973. 317 p.

TWILLEY, R. R. et al. Mangrove systems. In: HEYWOOD, V. H. (ed.). Global biodiversity assessment. Biodiversity and ecosystem function: ecosystem analyses. Cambridge: Cambridge University Press, 1995. p. 387-393. 